Pacific Journal of Mathematic 


\section{ON FUNCTIONS REPRESENTABLE AS A DIFFERENCE OF CONVEX FUNCTIONS}

\section{Philip HARTMAN}

1. Introduction. A function $f(x)$ defined on a convex $x$-set $D$ will be called a d.c. function on $D$ if there exists a pair of convex functions $F_{1}(x), F_{2}(x)$ on $D$ such that $f(x)$ is the difference

$$
f(x)=F_{1}(x)-F_{2}(x) .
$$

In this note, "convex" function means " continuous and convex" function. D.c. functions have been considered, for example, by Alexandroff [1]. E. G. Straus mentioned them in a lecture in Professor Beckenbach's seminar (and used the abbreviation "d.c.").

When $x$ is a real variable, so that $D$ is a (bounded or unbounded) interval, then $f(x)$ is a d.c. function if and only if $f$ has left and right derivatives (where these are meaningful) and these derivatives are of bounded variation on every closed bounded interval interior to $D$. Straus remarked that this fact implies that if $f_{1}(x), f_{2}(x)$ are d.c. functions of a real variable, then so are the product $f_{1}(x) f_{2}(x)$, the quotient $f_{1}(x) / f_{2}(x)$ when $f_{2}(x) \neq 0$, and the composite $f_{1}\left(f_{2}(x)\right)$ under suitable conditions on $f_{2}$. He raised the question whether or not this remark can be extended to cases where $x$ is a variable on a more general space. The object of this note is to give an affirmative answer to this question if $x$ is a point in a finite dimensional (Euclidean) space.

2. Local d.c. functions. Let $f(x)$ be defined on a convex $x$-set $D$. The function $f(x)$ will be said to be d.c. at a point $x_{0}$ of $D$ if there exists a convex neighborhood $U$ of $x_{0}$ such that $f(x)$ is d.c. on $U \cap D$. When $f(x)$ is d.c. at every point $x$ of $D$, it will be said to be locally d.c. on $D$.

(I) Let $D$ be a convex set in an m-dimensional Euclidean $x$-space and let $D$ be either open or closed. Let $f(x)$ be locally d.c. on $D$. Then $f(x)$ is d.c. on $D$.

While the proof of (I) cannot be generalized to the case where the $m$-dimensional $x$-space is replaced by a more general linear space, it will be clear that (II), below remains valid if the Euclidean $x$-space (but not the $y$-space) is replaced by a more general space.

Received February 10, 1959. This research was supported by the United States Air Force through the Air Force Office of Scientific Research of the Air Research and Development Command under contract No. AF 18 (603)-41. Reproduction in whole or in part is permitted for any purpose of the United States Government. 
(II) Let $x=\left(x^{1}, \cdots, x^{m}\right)$ and $y=\left(y^{1}, \cdots, y^{n}\right)$. Let $D$ and $E$ be convex sets in the $x$-and $y$-spaces, respectively; let $D$ be either open or closed and let $E$ be open. Let $g(y)$ be a d.c. function on $E$ and let $y^{j}=y^{j}(x)$ where $j=1, \cdots, n$, be d.c. functions on $D$ such that $y=y(x) \in E$ for $x \in D$. Then $f(x)=g(y(x))$ is locally d.c. on $D$.

This theorem is false (even for $n=m=1$ ) if the assumption that $E$ is open is omitted. In order to see this, let $x$ and $y$ be scalars, $g(y)=1-y^{1 / 2}(\leqq 1)$ on $E: 0 \leqq y<1$ and let $y=y(x)=\left|x-\frac{1}{2}\right|$ on $D: 0<x<1$. Since $f(x)=g(y(x))=1-\left|x-\frac{1}{2}\right|^{1 / 2}$ does not have finite left and right derivates at the interior point $x=\frac{1}{2}$, the function $f(x)$ is not d.c. at the point $x=\frac{1}{2}$.

It will be clear from the proof that (II) remains correct if the assumption that $E$ is open is replaced by the following assumption on $E$ and $g(y)$ : if $x_{0}$ is any point of $D$ and $y_{0}=y\left(x_{0}\right)$, let there exists a convex $y$-neighborhood $V$ of $y_{0}$ such that $g(y)$ satisfies a uniform Lipschitz condition on $V \cap E$. (This condition is always satisfied if $y_{0}$ is an interior point of $E$; cf., e.g., Lemma 3 below).

CoROLlary. Let $D$ be either an open or a closed convex set in the $\left(x^{1}, \cdots, x^{m}\right)$-space. Let $f_{1}(x), f_{2}(x)$ be d.c. functions on $D$. Then the product $f_{1}(x) f_{2}(x)$ and, if $f_{1}(x) \neq 0$, the quotient $f_{2}(x) \mid f_{1}(x)$ are d.c. functions on $D$.

The assertion concerning the product follows from (I) and (II) by choosing $y$ to be a binary vector $y=\left(y^{1}, y^{2}\right), g(y)=y^{1} y^{2}, E$ the $\left(y^{1}, y^{2}\right)$ plane and $y^{1}=f_{1}(x), y^{2}=f_{2}(x)$. Thus $f(x) \equiv g(y(x))=f_{1}(x) f_{2}(x)$. Note that $g(y)=\frac{1}{2}\left(y^{1}+y^{2}\right)^{2}-\frac{1}{2}\left(\left(y^{1}\right)^{2}+\left(y^{2}\right)^{2}\right)$ is a d.c. function on $E$.

In the assertion concerning the quotient, it can be supposed that $f_{2}(x) \equiv 1$ and that $f_{1}(x)>0$. Let $y$ be a scalar, $g(y) \equiv 1 / y$ on $E: y>0$ and $y=f_{1}(x)$ on $D$. Thus $g(y)$ is convex on $E$ and $f(x) \equiv g(y(x))=1 / f_{1}(x)$.

3. Preliminary lemmas. It will be convenient to state some simple lemmas before proceeding to the proofs of (I) and (II). The proofs of these lemmas will be indicated for the sake of completeness.

In what follows, $x=\left(x^{1}, \cdots, x^{m}\right)$ is an $m$-dimensional Euclidean vector and $|x|$ is its length. $D$ is a convex set in the $x$-space.

Lemma 1. Let $D$ be either an open or a closed convex set having interior points. Let $x=x_{0}$ be a point of $D$ and $U$ a convex neighborhood of $x_{0}$. Let $F(x)$ be a convex function on $D \cap U$. Then there exists a neighborhood $U_{1}$ of $x_{0}$ and a function $F_{1}(x)$ defined and convex on $D$ such that $F(x) \equiv F_{1}(x)$ on $D \cap U_{1}$.

In order to see this, let $U_{2}$ be a small sphere $\left|x-x_{0}\right|<r$ such that $F(x)$ is bounded on the closure of $D \cap U_{2}$. Let $G(x)=K\left|x-x_{0}\right|+$ 
$F\left(x_{0}\right)-1$, where $K$ is a positive constant, chosen so large that $G(x)>$ $F(x)+1>F(x)$ on the portion of the boundary of $U_{2}$ interior to $D$. Clearly $G(x)<F(x)$ holds for $x=x_{0}$, hence, for $x$ on $D \cap U_{1}$ if $U_{1}$ is a suitably chosen neighborhood of $x_{0}$. If $x \in D$, define $F_{1}(x)$ to be $\max (F(x), G(x))$ or $G(x)$ according as $x$ is or is not in $U_{2} \cap D$. Since $\max (F(x), G(x))$ is convex on $U_{2} \cap D$ and $\max (F(x), G(x)) \equiv G(x)$ for $x$ in a vicinity (relative to $D$ ) of the boundary of $U_{2}$ in $D$, it follows that $F_{1}(x)$ is convex on $D$. Finally, $F_{1}(x) \equiv \max (F(x), G(x))=F(x)$ for $x \in U_{1} \cap D$.

Lemma 2. Let $D$ be a closed, bounded convex set having $x=0$ as an interior point. There exists a function $h(x)$ defined and convex for all $x$ such that $h(x) \leqq 1$ or $h(x)>1$ according as $x \in D$ or $x \notin D$.

In fact, $h(x)$ can also be chosen so as to satisfy $h(x)>0$ for $x \neq=0$ and $h(c x)=c h(x)$ for $c>0$. This function is then the supporting function of the polar convex set of $D$; Minkowski, cf. [2], §4. The function $h(x)$ is given by 0 or $|x| \rho^{-1}(x /|x|)$ according as $x=0$ or $x \neq 0$, where, if $u$ is a unit vector, $\rho(u)$ is the distance from $x=0$ to the point where the ray $x=t u, t>0$ meets the boundary of $D$.

Lemma 3. Let $D$ be a closed, bounded convex set having interior points and $D_{1}$ a closed convex set interior to $D$. Let $F(x)$ be a convex function on $D$. Then $F(x)$ satisfies a uniform Lipschitz condition on $D_{1}$.

In fact, if $d>0$ is the distance between the boundaries of $D$ and $D_{1}$ and if $|F(x)| \leqq M$ on $D$, then $\left|F\left(x_{1}\right)-F\left(x_{2}\right)\right| \leqq 2 M\left|x_{1}-x_{2}\right| / d$ for $x_{1}, x_{2} \in D_{1}$. This inequality follows from the fact that $F(x)$ is convex on the intersection of $D$ and the line through $x_{1}$ and $x_{2}$.

4. Proof of (I). The proof will be given for the case of an open convex set $D$. It will be clear from the proof and from Lemma 1 how the proof should be modified for the case of a closed $D$.

To every point $x_{0}$ of $D$, there is a neighborhood $U=U\left(x_{0}\right)$, say $U$ : $\left|x-x_{0}\right|<r\left(x_{0}\right)$, contained in $D$ such that $f(x)$ is d.c. on $U$; that is, there exists a convex function $F(x)=F\left(x, x_{0}\right)$ such that $f(x)+F\left(x, x_{0}\right)$ is a convex function of $x$ on $U\left(x_{0}\right)$. In view of Lemma 1 , it can be supposed (by decreasing $r\left(x_{0}\right)$, if necessary) that $F\left(x, x_{0}\right)$ is defined and convex on $D$ (although, of course, $f+F$ is convex only on $U$ ).

Let $D_{1}$ be a compact, convex subset of $D$. Then $D_{1}$ can be covered by a finite number of the neighborhoods $U\left(x_{1}\right), \cdots, U\left(x_{k}\right)$. Put $F(x)=$ $F\left(x, x_{1}\right)+\cdots+F\left(x, x_{k}\right)$, so that $F(x)$ is defined and convex on $D$. Since $f(x)+F\left(x, x_{j}\right)$ is convex on $U\left(x_{j}\right)$, so is $f(x)+F(x)=f(x)+F\left(x, x_{j}\right)+$ 
$\sum_{i \neq j} F\left(x, x_{i}\right)$. Hence $f+F$ is convex on $D_{1}$.

Thus there exists a sequence of open, bounded convex sets $D_{1}, D_{2}, \ldots$ with the properties that the closure of $D_{j}$ is contained in $D_{j+i}, D=\bigcup D_{j}$, and to each $D_{j}$ there corresponds a function $F_{j}(x)$ defined and convex on $D$ such that $f(x)+F_{j}(x)$ is convex on $D_{j}$.

Introduce a sequence of closed convex sets $C^{1}, C^{2}, \cdots$ such that $C^{1} \subset D_{1} \subset C^{2} \subset D_{2} \subset \cdots$. In particular, $D=\mathrm{U} C^{j}$.

It will be shown that there is a function $G_{1}(x)$ with the properties that

(i) $G_{1}(x)$ is defined and convex on $D$,

(ii) $f(x)+G_{1}(x)$ is convex on $D_{2}$, and

(iii) $G_{1}(x) \equiv F_{1}(x)$ on $C^{1}$.

If this is granted for the moment, the proof of (I) can be completed as follows: If $G_{1}, \cdots, G_{k-1}$ have been constructed, let $G_{k}$ be a function defined and convex on $D$ such that $f+G_{k}$ is convex on $D_{k+1}$ and $G_{k} \equiv$ $G_{k-1}$ on $C^{k}$. Then $F(x)=\lim G_{k}(x)$ exists uniformly on compact subsets of $D$; in fact, $F(x) \equiv G_{j}(x)$ on $C^{k}$ for all $j \geqq k$. Hence, $F(x)$ is defined and convex on $D$. Since $f(x)+F(x)$ is convex on $C^{k}, k=1,2, \cdots$, it is convex on $D$; that is, $f$ is a d.c. function on $D$.

Thus, in order to complete the proof of (I), it remains to construct a $G_{1}(x)$ with the properties (i) - (iii). Let $k>0$ be a constant so large that $F_{2}(x)-k \leqq F_{1}(x)$ for $x \in C^{1}$. Without loss of generality, it can be supposed that $x=0$ is an interior point of $C^{1}$. Let $h(x)$ be the function given by Lemma 2 when $D$ there is replaced by $C^{1}$. Put $H(x)=0$ or $H(x)=K[h(x)-1]$ according as $x \in C^{1}$ or $x \notin C^{1}$, where $K>0$ is a constant. Thus $H(x)$ is defined and convex for all $x$ and $H(x) \equiv 0$ on $C^{1}$. In particular,

$$
F_{2}(x)-k+H(x) \leqq F_{1}(x) \text { for } x \in C^{1} .
$$

Choose $K$ so large that

$$
F_{2}(x)-k+H(x)>F_{1}^{\prime}(x) \text { on } D_{1}^{\prime}
$$

the boundary of $D_{1}$. This is possible since $h(x)-1>0$ for $x \notin C^{1}$.

Define $G_{1}(x)$ as follows:

$$
\begin{aligned}
& G_{1}(x)=\max \left(F_{1}(x), F_{2}(x)-k+H(x)\right) \text { for } x \in D_{1}, \\
& G_{1}(x)=F_{2}(x)-k+H(x) \text { for } x \in D-D_{1},
\end{aligned}
$$

where $D-D_{1}$ is the set of points in $D$, not in $D_{1}$.

Clearly, (2) and the first part of (4) imply property (iii),

$$
G_{1}(x)=F_{1}(x) \text { if } x \in C^{1},
$$

and (3) implies that 


$$
G_{1}(x)=F_{2}(x)-k+H(x) \text { for } x \text { on and near } D_{1}^{\prime},
$$

the boundary of $D_{1}$.

By the first part of (4), $G_{1}(x)$ is convex on $D_{1}$. By the last part of (4) and by (6), $G_{1}(x)$ is convex in a vicinity of every point of $D-D_{1}$. Hence, $G_{1}(x)$ has property (i), that is $G_{1}(x)$ is convex on $D$.

Since $f(x)+F_{1}(x)$ is convex on $D_{1}$ and $f(x)+F_{2}(x)$, hence $f(x)+$ $F_{2}(x)-k+H(x)$ is convex on $D_{2} \supset D_{1}$, it follows that, on $D_{1}$, the function

$$
f(x)+G_{1}(x)=\max \left(f+F_{1}, f+F_{2}-k+H\right)
$$

is convex. It also follows from the last part of (4) and from (6) that $f+G_{1}$ is convex in a vicinity of every point of $D_{2}-D_{1}$. Hence $G_{1}$ has property (ii), that is, $f+G_{1}$ is convex on $D_{2}$. This completes the proof of (I).

5. Proof of (II). Without loss of generality, it can be supposed that $g(y)$ is convex on $E$.

Since $y^{j}(x)$ is a d.c. function on $D$, there exists a convex function $F(x)$ on $D$ such that

$$
\pm y^{j}(x)+F(x) \text { are convex on } D .
$$

The function $F(x)=F(x, j, \pm)$ can be assumed to be independent of $j$, where $j=1, \cdots, n$, and of \pm ; for otherwise it can be replaced by $\sum_{j} F(x, j,+)+\sum_{j} F(x, j,-)$.

Let $x=x_{0}$ be a point of $D$ and $y_{0}=y\left(x_{0}\right)$. Let $V$ be a convex neighborhood of $y_{0}$ such that $g$ satisfies a uniform Lipschitz condition

$$
\left|g\left(y_{1}\right)-g\left(y_{2}\right)\right| \leqq M\left|y_{1}-y_{2}\right|
$$

on $V$; cf. Lemma 3 . Let $U$ be a neighborhood of $x_{0}$ such that $y(x) \in V$ for $x \in U \cap D$. It will be shown that

$$
f(x)+3 n M F(x) \text { is convex on } D \cap U,
$$

so that $f$ is d.c. at $x=x_{0}$.

It is clear that there is no loss of generality in assuming that $g(y)$ has continuous partial derivatives satisfying

$$
|\partial g(y)| \partial y^{j} \mid \leqq M \text { for } j=1, \cdots, n \text { and } y \in V .
$$

For otherwise, $g$ can be approximated by such functions.

In what follows, only $x$ in $D \cap U$ and $y \in V$ occur. Let $x=x(s)$, where $s$ is a real variable on some interval, be an arc-length parametrization of a line segment in $D \cap U$. The assertion (9) follows if it is shown that $e(s)+3 n M F(x(s))$, where $e(s)=f(x(s))$ is a convex function 
of $s$. It is clear that $e(s)$ has left and right derivatives (whenever these are meaningful). Let $e^{\prime}(s)$ denote a left or a right derivate of $e(s)$ and $F^{\prime}(x(s)), y^{j^{\prime}}(x(s))$ the corresponding derivates of $F(x(s)), y^{j}(x(s))$. Let $\Delta s>0$, then $e(s)+3 n M F(x(s))$ is convex if and only if $\Delta e^{\prime}+3 n M \Delta F^{\prime} \geqq 0$, where $\Delta e^{\prime}=e^{\prime}(s+\Delta s)-e^{\prime}(s)$ and $\Delta F^{\prime}=F^{\prime}(x(s+\Delta s))-F^{\prime}(x(s))$.

By the definition of $e$,

$$
e^{\prime}=\sum\left(\partial g / \partial y^{\prime}\right) y^{\prime \prime}
$$

Hence,

$$
\Delta e^{\prime}(s)=\sum \Delta\left(\partial g / \partial y^{j}\right) y_{0}^{j^{\prime}}+\sum\left(\partial g / \partial y^{j}\right)_{1} \Delta_{y}^{j^{\prime}},
$$

where $y_{0}^{j^{\prime}}=y^{j^{\prime}}(x(s))$ and $\left(\partial g / \partial y^{j}\right)_{1}$ is the value of $\partial g / \partial y^{j}$ at $y=y(x(s+\Delta s))$.

The usual proofs of the mean value theorem of differential calculus (via Rolle's theorem) imply the existence of a $0=\theta_{j}, 0<\theta_{j}<1$, such that

$$
\Delta_{y}^{j} / \Delta s=y_{\theta}^{y^{\prime}},
$$

where $y_{\theta}^{j^{\prime}}$ is a number between the left and right derivates of $y^{j}(x(s))$ at the $s$-point $s+\theta_{j} \Delta s$. By (13), the equation (12) can be written as

$$
\begin{aligned}
\Delta e^{\prime}=\sum\left(\Delta \partial g / \partial y^{j}\right)\left(\Delta y^{j} / \Delta s\right) & +\sum\left(\Delta \partial g / \partial y^{j}\right)\left(y_{0}^{j^{\prime}}-y_{\theta}^{j^{\prime}}\right) \\
& \left.+\sum\left(\partial g / \partial y^{j}\right)_{1} \Delta y^{j^{\prime}}\right) .
\end{aligned}
$$

By (7),

$$
\left|\Delta y^{\jmath^{\prime}}\right| \leqq \Delta F^{\prime} \text { and }\left|y_{0}^{y^{\prime}}-y_{\theta}^{j^{\prime}}\right| \leqq F_{\theta}^{\prime}-F_{0}^{\prime} \leqq \Delta F^{\prime},
$$

where $F_{\theta}^{\prime}$ is the right derivate of $F(x(s))$ at the $s$-point $s+\theta_{j} \Delta s(<s+\Delta s)$. Since $g(y)$ is convex, the first term on the right of (14) in non-negative. Hence (10) and (11) give

$$
\Delta e^{\prime} \geqq 0-2 n M \Delta F^{\prime}-M n \Delta F^{\prime},
$$

so that $e(s)+3 n M F(x(s))$ is convex. This proves (II).

6. "Minimal" convex functions. Let $f(x)$ be d.c. on the unit sphere $|x|<1$, so that there exist functions $F(x)$ on $|x|<1$ such that

$$
F(x) \text { and } f(x)+F(x) \text { are convex on }|x|<1 \text {. }
$$

The function $F(x)$ can be chosen so as to satisfy the normalization

$$
F(0)=0 \text { and } F(x) \geqq 0 .
$$

If $x$ is a real variable, there exists a "least" $F(x)$, say $F_{m}(x)$, satisfying (16), (17) in the sense that (16), (17) hold for $F=F_{m}$ and (16), (17) imply 


$$
F_{m}(x) \leqq F(x) \text { on }|x|<1 .
$$

In fact, $F_{m}(x)$ can be obtained as follows: A (left or right) derivative $f^{\prime}(x)$ of $f(x)$ is of bounded variation on every interval $|x| \leqq a<1$ and so $f^{\prime}(x)$ can be written as $f^{\prime}(x)=P(x)-N(x)$, where $P(x), N(x)$ are the positive, negative variation of $f^{\prime}$ on the interval between 0 and $x$, say, with the normalization $N(0)=0$. In particular, $P$ and $N$ are nondecreasing on $|x|<1$. In this case, $F_{m}(x)$ is given by

$$
F_{m}(x)=\int_{0}^{x} N(x) d x \text {. }
$$

On the other hand, if $x$ is a vector, there need not exist a least $F=F_{m}(x)$. In order to see this, let $x$ be a binary vector and write $(x, y)$ instead of $x$. Let $f(x, y)=x y$. If $\varepsilon>0, F(x, y)=\frac{1}{2}\left(\varepsilon x^{2}+y^{2} / \varepsilon\right)$ satisfies (16), (17). If a least $F=F_{m}$ exists, then $0 \leqq F_{m}(x, y) \leqq \frac{1}{2}\left(\varepsilon x^{2}+\right.$ $\left.y^{2} / \varepsilon\right)$. In particular, $0 \leqq F_{m}(x, 0) \leqq \varepsilon x^{2}$, and, therefore, $F_{m}(x, 0) \equiv 0$. Similarly, $F_{m}(0, y) \equiv 0$. But since $F_{m}$ is convex, it follows that $F_{m} \equiv 0$. This contradicts the case $F=F_{m}$ of (16) and so, a least $F=F_{m}$ does no exist.

Although a "least" $F$ need not exist, it follows from Zorn's lemma that " minimal" $F$ 's do exist.

\section{REFERENCES}

1. A. D. Alexandroff, Surfaces represented by the difference of convex functions, Doklady Akademii Nauk SSSR, (N.S.) 72 (1950), 613-616.

2. T. Bonnesen and W. Fenchel, Theorie der konvexen Korper, New York (1948).

THE JOHNS HOPKINS INIVERSITY 



\section{PACIFIC JOURNAL OF MATHEMATICS}

\section{EDITORS}

\section{David Gilbarg}

Stanford University Stanford, California

\section{R. A. Beaumont}

University of Washington

Seattle 5, Washington

\section{A. L. Whiteman}

University of Southern California Los Angeles 7, California

L. J. Paige

University of California

Los Angeles 24, California

\section{ASSOCIATE EDITORS}

E. F. BECKENBACH
C. E. BURGESS
E. HEWITT
A. HORN

A. HORN

\author{
V. GANAPATHY IYER \\ R. D. JAMES \\ M. S. KNEBELMAN \\ L. NACHBIN
}

I. NIVEN

T. G. OSTROM

H. L. ROYDEN

M. M. SCHIFFER
E. G. STRAUS

G. SZEKERES

F. WOLF

K. YOSIDA

\section{SUPPORTING INSTITUTIONS}

\author{
UNIVERSITY OF BRITISH COLUMBIA \\ CALIFORNIA INSTITUTE OF TECHNOLOGY \\ UNIVERSITY OF CALIFORNIA \\ MONTANA STATE UNIVERSITY \\ UNIVERSITY OF NEVADA \\ OREGON STATE COLLEGE \\ UNIVERSITY OF OREGON \\ OSAKA UNIVERSITY \\ UNIVERSITY OF SOUTHERN CALIFORNIA
}

\author{
STANFORD UNIVERSITY \\ UNIVERSITY OF TOKYO \\ UNIVERSITY OF UTAH \\ WASHINGTON STATE COLLEGE \\ UNIVERSITY OF WASHINGTON \\ * * * * \\ AMERICAN MATHEMATICAL SOCIETY \\ CALIFORNIA RESEARCH CORPORATION \\ HUGHES AIRCRAFT COMPANY \\ SPACE TECHNOLOGY LABORATORIES
}

Mathematical papers intended for publication in the Pacific Journal of Mathematics should be typewritten (double spaced), and the author should keep a complete copy. Manuscripts may be sent to any one of the four editors. All other communications to the editors should be addressed to the managing editor, L. J. Paige at the University of California, Los Angeles 24, California.

50 reprints per author of each article are furnished free of charge; additional copies may be obtained at cost in multiples of 50 .

The Pacific Journal of Mathematics is published quarterly, in March, June, September, and December. The price per volume (4 numbers) is $\$ 12.00$; single issues, $\$ 3.50$. Back numbers are available. Special price to individual faculty members of supporting institutions and to individual members of the American Mathematical Society: $\$ 4.00$ per volume; single issues, $\$ 1.25$.

Subscriptions, orders for back numbers, and changes of address should be sent to Pacific Journal of Mathematics, 2120 Oxford Street, Berkeley 4, California.

Printed at Kokusai Bunken Insatsusha (International Academic Printing Co., Ltd.), No. 6, 2-chome, Fujimi-cho, Chiyoda-ku, Tokyo, Japan.

PUBLISHED BY PACIFIC IOURNAL OF MATHEMATICS, A NON-PROFIT CORPORATION

The Supporting Institutions listed above contribute to the cost of publication of this Journal, but they are not owners or publishers and have no responsibility for its content or policies. 


\section{Pacific Journal of Mathematics}

\section{Vol. 9, No. $3 \quad$ July, 1959}

Errett Albert Bishop, A minimal boundary for function algebras . . . . . . . . . . . . 629

John W. Brace, The topology of almost uniform convergence . . . . . . . . . . . . 643

Cecil Edmund Burgess, Chainable continua and indecomposability .......... 653

L. Carlitz, Multiplication formulas for products of Bernoulli and Euler

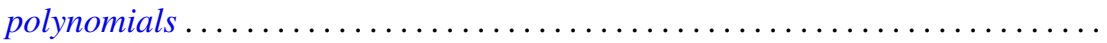

Eckford Cohen, A class of residue systems (mod $r$ ) and related arithmetical

functions. II. Higher dimensional analogues ....................

Shaul Foguel, Boolean algebras of projections of finite multiplicity . . . . . . . . . .

Richard Robinson Goldberg, Averages of Fourier coefficients .................

Seymour Goldberg, Ranges and inverses of perturbed linear operators .

Philip Hartman, On functions representable as a difference of convex functions ....

Milton Vernon Johns, Jr. and Ronald Pyke, On conditional expectation and

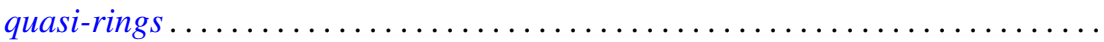

Robert Jacob Koch, Arcs in partially ordered spaces ....................

Gregers Louis Krabbe, A space of multipliers of type $L^{p}(-\infty, \infty) \ldots \ldots \ldots \ldots$

John W. Lamperti and Patrick Colonel Suppes, Chains of infinite order and their

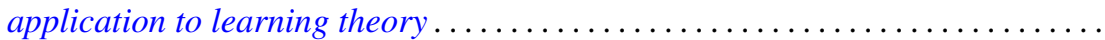

Edith Hirsch Luchins, On radicals and continuity of homomorphisms into Banach

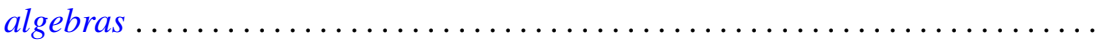

T. M. MacRobert, Multiplication formulae for the E-functions functions of their parameters.

Michael Bahir Maschler, Classes of minimal and representative domains and their kernel functions.

William Schumacher Massey, On the imbeddability of the real projective spaces in Euclidean space.

Thomas Wilson Mullikin, Semi-groups of class $\left(C_{0}\right)$ in $L_{p}$ determined by parabolic differential equations

Steven Orey, Recurrent Markov chains

Ernest Tilden Parker, On quadruply transitive groups ........ . .

Calvin R. Putnam, On Toeplitz matrices, absolute continuity, and unitary

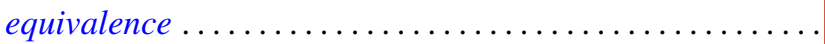

Helmut Heinrich Schaefer, On nonlinear positive operators.

Robert Seall and Marion Wetzel, Some connections between continued fractions and convex sets

Robert Steinberg, Variations on a theme of Chevalley

Olga Taussky and Hans Zassenhaus, On the similarity transformation between a

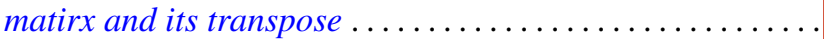

Emery Thomas, The suspension of the generalized Pontrjagin cohomology

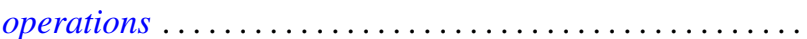

Joseph L. Ullman, On Tchebycheff polynomials ..................... 913

Richard Steven Varga, Orderings of the successive overrelaxation scheme ........ 925

Orlando Eugenio Villamayor, Sr., On weak dimension of algebras . 